\title{
Design of Air Pump System Using Bond Graph and Genetic Programming Method
}

\author{
Kisung Seo \\ Dept. of Electronics Engineering \\ Seokyeong University \\ Seoul 136-749, KOREA \\ ksseo@skuniv.ac.kr
}

\author{
Erik D. Goodman \\ Dept. of ECE \\ Michigan State University \\ East Lansing, MI 48824 USA \\ goodman@egr.msu.edu
}

\author{
Ronald C. Rosenberg \\ Dept. of Mechanical Engineering, \\ Michigan State University \\ East Lansing, MI 48824 USA \\ rosenber@egr.msu.edu
}

\begin{abstract}
This paper introduces a redesign method for an air pump system using bond graphs and genetic programming to maximize outflow subject to a constraint specifying maximum power consumption. The redesign process can alter the topological connections among components and can introduce additional components. The air pump system is a mixed-domain system that includes electromagnetic, mechanical and pneumatic elements. Bond graphs are domain independent, allow free composition, and are efficient for classification and analysis of models. Genetic programming is well recognized as a powerful tool for openended search. The combination of these two powerful methods, $\mathrm{BG} / \mathrm{GP}$, was applied for redesign of an air pump system.
\end{abstract}

\section{Categories and Subject Descriptors}

J.6 [Computer-Aided Engineering]: Computer-aided design (CAD)

\section{General Terms}

Algorithms, Design

\section{Keywords}

Automated Design, Bond Graph, Genetic Programming, Air Pump

\section{INTRODUCTION}

A multi-domain dynamic system includes a mixture of, for example, electrical, mechanical, hydraulic, pneumatic, and/or thermal components. Multi-energy domain design is difficult because such systems tend to be complex and most current simulation tools operate over only a single domain. In order to automate design of multi-domain systems, such as mechatronic systems, a new approach is required[1].

The Bond Graph / Genetic Programming (BG/GP) design methodology[2] has been developed to overcome limitations of single-domain design approaches and enable open-ended, automatic search, based on the combination of these tools; it has been tested and has worked efficiently for a few applications[3,4].

Copyright is held by the author/owner(s).

GECCO05, June 25-29, 2005, Washington, DC, USA.

ACM 1-59593-010-8/05/0006.
In this paper, BG/GP is applied to design an air pump that consists of mixed-energy-domain elements, seeking to maximize outflow subject to a constraint specifying maximum power consumption. Section 2 explains the air pump system. Section 3 describes the Bond Graph / Genetic Programming approach. Sections 4 and 5 describe the redesign problem of the air pump system and present design results. Section 6 concludes the paper.

\section{AIR PUMP SYSTEM}

The air pump system[5], is a mixed-energy-domain system, because it includes electric, electromagnetic, mechanical and pneumatic elements; a unified approach is required to design it. A schematic of an initial air pump design, used as a starting point in this redesign study, is presented in Figure 1. It is a vibratory pump in which an electromagnetic circuit drives a small permanent magnet attached to a pivoted lever that, in turn, drives a rubber bellows pump. The bellows pump has rubber check valves and delivers a small flow of air. The basic structure of the air pump consists of the cascaded arrangement of three coupled subsystems: the electromagnetic actuator, the lever, and the air bellows.

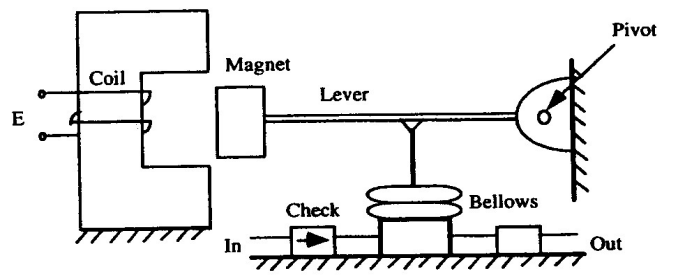

Figure 1. Schematic of initial air pump design

\section{BG/GP DESIGN PROCESS}

Bond graph modeling is a powerful method that enables a unified approach to the analysis, synthesis and evaluation of dynamic systems. It represents the common energy processes of multidomain systems - electrical, mechanical, fluid, and thermal systems - in one graphical notation [6,7].

The overall design procedure is shown in Figure 2. The designer sets the design context by specifying an embryo bond graph model. Parameters for the GP search process must be set to control both the generation phase (yielding an initial population of candidate solutions in the form of GP trees) and the evolution phase. At each stage of evolution, each of the candidates is evaluated and assigned a fitness value. The evolution phase is guided by the statistics of the selection and evolution operators. 
The evolution process terminates when fitness or effort conditions are met. The result is reported as a bond graph (or set of them) with the highest fitness rating(s).

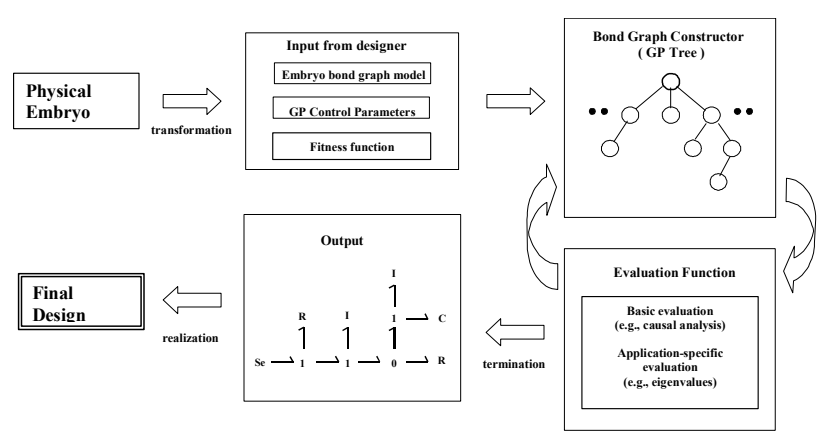

Figure 2. The BG/GP design procedure

\section{AIR PUMP REDESIGN}

The average peak value of outflow for the original air pump (Figure 1) is approximately $2 \times 10^{-4} \mathrm{~m}^{3} / \mathrm{sec}$ and an improved design found in our earlier work performed $13.6 \%$ better than the original one [2]. The ultimate objective of the pump redesign is to maximize outflow subject to a constraint specifying maximum power consumption. The new design reported here is represented in Figure 3. BG/GP evolved three new components at modifiable sites of the embryo (two $\mathrm{C}$, one $\mathrm{TF}$, appearing between the two dashed vertical lines in Figure 3). Figure 4 displays the muchimproved outflow of this design variant.

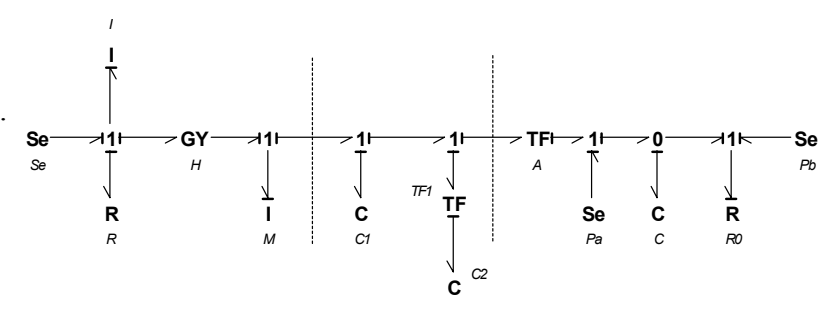

Figure 3. Evolved bond graph redesign for air pump

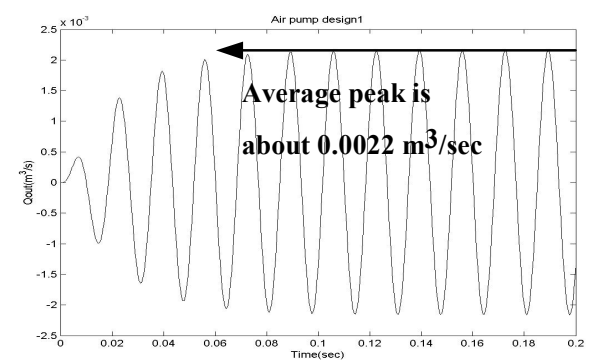

Figure 4. Outflow of redesigned model for air pump

The average peak value of outflow is approximately $2.2 \times 10^{-3}$ $\mathrm{m}^{3} / \mathrm{sec}$, which is almost 9 times better than the original model; the power consumption, which was not constrained in this study, is also higher, but by a much smaller factor. Physical realization of the design is shown in Figure 5.

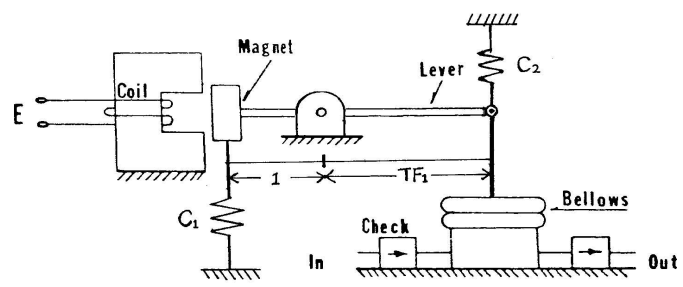

Figure 5. Physical realization of design variant

\section{CONCLUSION}

This paper has introduced a conceptual or topological redesign of an air pump system using the BG/GP design method. The domain of the air pump system includes electromagnetic and pneumatic fields. The modeling, analysis and synthesis are performed using a unified (multi-domain) approach. A physically realizable design candidate for the air pump system, with an output flow much higher than that of the original model, is obtained. This, in turn, offers promise that much more complex multi-domain systems with more complex performance specifications can be designed efficiently. The next step in the pump redesign process will be to specify a maximum power constraint and then seek designs that maximize the outflow produced subject to that power constraint. Reduction of the size of the embryo might also allow more radically different redesigns to emerge.

\section{REFERENCES}

[1] Coelingh, E., T. de Vries, J. Amerongen, “Automated Performance Assessment of Mechatronic Motion Systems during the Conceptual Design Stage," Proc. $3^{\text {rd }}$ Int'l Conf. on Adv. Mechatronics, Okayama, Japan, 1998.

[2] Seo K., Goodman, E., Rosenberg, R. C.: First Steps toward Automated Design of Mechatronic Systems Using Bond Graphs and Genetic Programming. Proc. Genetic and Evolutionary Computation Conference, GECCO-2001, San Francisco (2001) 189

[3] Seo K., Hu, J., Fan, Z., Goodman E. D., Rosenberg R. C.: Automated Design Approaches for Multi-Domain Dynamic Systems Using Bond Graphs and Genetic Programming. Int. Jour. of Computers, Systems and Signals, 3(1). (2002) 55-70

[4] Fan Z., Seo, K., Rosenberg R. C., Hu J., Goodman E. D.: Exploring Multiple Design Topologies using Genetic Programming and Bond Graphs. Proceedings of the Genetic and Evolutionary Computation Conference, GECCO-2002, New York (2002) 1073-1080.

[5] Tay E., Flowers W. and Barrus J., “Automated Generation and Analysis of Dynamic System Designs," Research in Engineering Design, vol 10, 1998, 15-29.

[6] Karnopp, D. C., Rosenberg R. C., Margolis, D. L., System Dynamics, A Unified Approach, $3^{\text {nd }}$ ed., John Wiley \& Sons (2000)

[7] Sharpe, J. E., Bracewell R. H. " The Use of Bond Graph Reasoning for the Design of Interdisciplinary Schemes," 1995 International Conference on Bond Graph Modeling and Simulation, 1995, 116-121 